\title{
Synthesis of 4-cyclo(propyl- and butyl)-1-ethylpyridinium bromides and calculation of their proton and carbon chemical shifts
}

\author{
Olegas Eicher-Lorka*, Zenonas Kuodis, Algirdas Matijoška, and Albertas Rutavičius \\ Institute of Chemistry, Goštauto Str., Vilnius, LT-01108, Lithuania \\ E-mail:lorka@ktl.mii.lt
}

\begin{abstract}
New 4-cyclo(propyl- and butyl)pyridinium salts have been prepared by alkylation of 4picolinium salt with $\alpha, \omega$-dibromoalkanes under phase-transfer catalysis conditions. The influence of the nature and the catalysis concentration was investigated. The experimentally determined ${ }^{1} \mathrm{H}$ and ${ }^{13} \mathrm{C}$ NMR chemical shifts were compared with the values calculated using the GIAO/DFT and HF approach in gas-phase and/or in methanol by using the IEF-PCM method.
\end{abstract}

Keywords: Cyclopropylpyridinium, cyclobutylpyridinium, phase-transfer catalysis, NMR chemical shift calculation.

\section{Introduction}

The cyclopropyl group is an increasingly common structural motif in pharmaceutically active molecules and is frequently included as a substituent in structure-activity relationship studies. ${ }^{1}$ Either the cyclopropyl or the cyclobutyl is a relatively highly reactive group and are frequently used as an intermediate in organic synthesis. ${ }^{2,3}$ Compounds containing the cyclopropylpyridine moiety exhibit antibiotic, ${ }^{4}$ antidepressant and anticancer activity. ${ }^{5}$ The cyclopropylpyridines can be prepared by reaction of the appropriate vinylpyridine with diazocompounds ${ }^{6,7}$ or dimethylsulfoniummethylide, ${ }^{8}$ treatment of 4-picolyl chloride with an acrylate ester or acrylonitrile and sodium hydride, Error! Bookmark not defined. presence of magnesium organic compounds ${ }^{9}$ and by Suzuki coupling reaction of cyclopropylboronic acid with pyridine bromides. ${ }^{10}$ These methods suffer from the disadvantages such as many steps reactions ${ }^{\text {Error! Bookmark not defined. }}$ and costly or rare reagents. ${ }^{\text {Error! Bookmark not }}$ defined.-Error! Bookmark not defined. Alkylation with dihalogenides has been applied to many compounds with activated methylene group. ${ }^{11}$ This type of cyclization reaction allows synthesizing compounds with cycles of three and more methylene groups. ${ }^{12}$ Sometimes base-induced cyclization reaction was carried out at phase-transfer catalysis (PTC) condition in liquid-liquid two phase system. ${ }^{13}$ Previously, we reported the study of the alkylation of 4-methylpyridinium 
salts with monohalogenalkanes. ${ }^{14}$ We have found that the alkylation using $\mathrm{K}_{2} \mathrm{CO}_{3}$ as the base and acetonitrile as solvent with PT catalysts leads to mono-, di- and tri- alkylated products in good yields. Herein, we report a simple one-pot synthesis of 4-cyclopropyl- and cyclobutylpyridinium salts from $\alpha, \omega$-dibromoalkanes and 4-methylpyridinium salt.

The molecular structure and conformation of cyclopropylbenzene and 4-cyclopropylpyridine were the subject of experimental and theoretical studies. ${ }^{15,16}$ Experimental NMR spectroscopy data combined with molecular modelling at the density functional level of theory can nowadays provide an accurate description of the chemical structure and conformational behavior of organic substances. In this work, we applied a molecular modelling protocol to new synthesized compounds. The experimentally determined proton and carbon chemical shifts $(\delta / \mathrm{ppm})$ were compared with calculated values using the GIAO/DFT and HF approach.

\section{Computational methods}

Structures 3a-c first were fully optimized at the B3LYP/6-311++G(2d,p) level without solvent or in methanol by using the IEF-PCM method in Gaussian $03 \mathrm{~W} .{ }^{17}$ The torsional potential function about the $\mathrm{C}-\mathrm{C}$ bond connecting the cyclo(propyl and butyl) and pyridinium groups $(\alpha)$ has been calculated at $30^{\circ}$ interval with full geometric optimization at the each point. The bisected forms of cyclo(propyl and butyl)pyridiniums are preferred over other forms (Figure 1). All compounds may assume two different bisected conformations, with very small differences in energy, due to ethyl group orientation over the pyridinium ring. The general shape of the potential curves of compounds 3a-c are similar, with a global minimum for the bisected form and only 4cyclobutylpyridinium have another minimum for the perpendicular forms as and 4cyclopropylpyridine and cyclopropylbenzene (Figure 2).

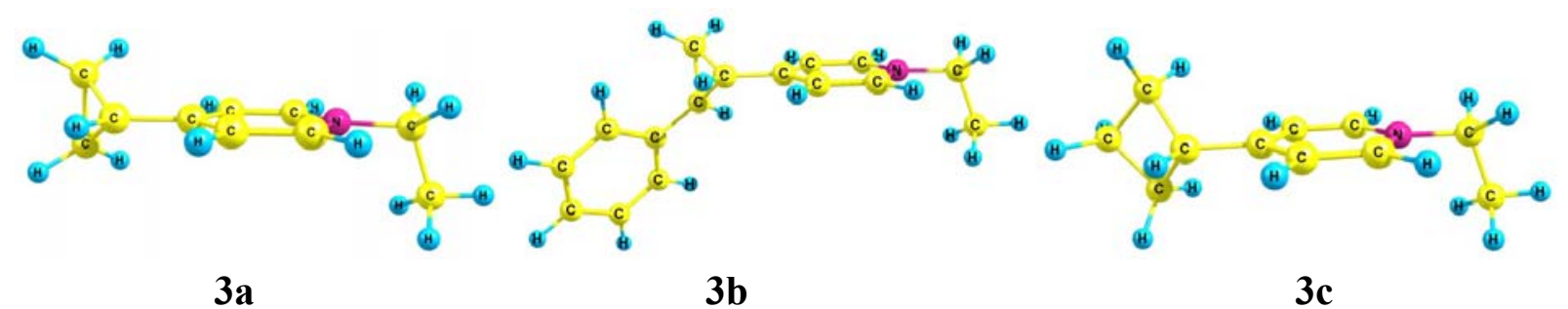

Figure 1. Bisected forms of compounds 3a-c (visualized with ChemCraft program). ${ }^{18}$ 


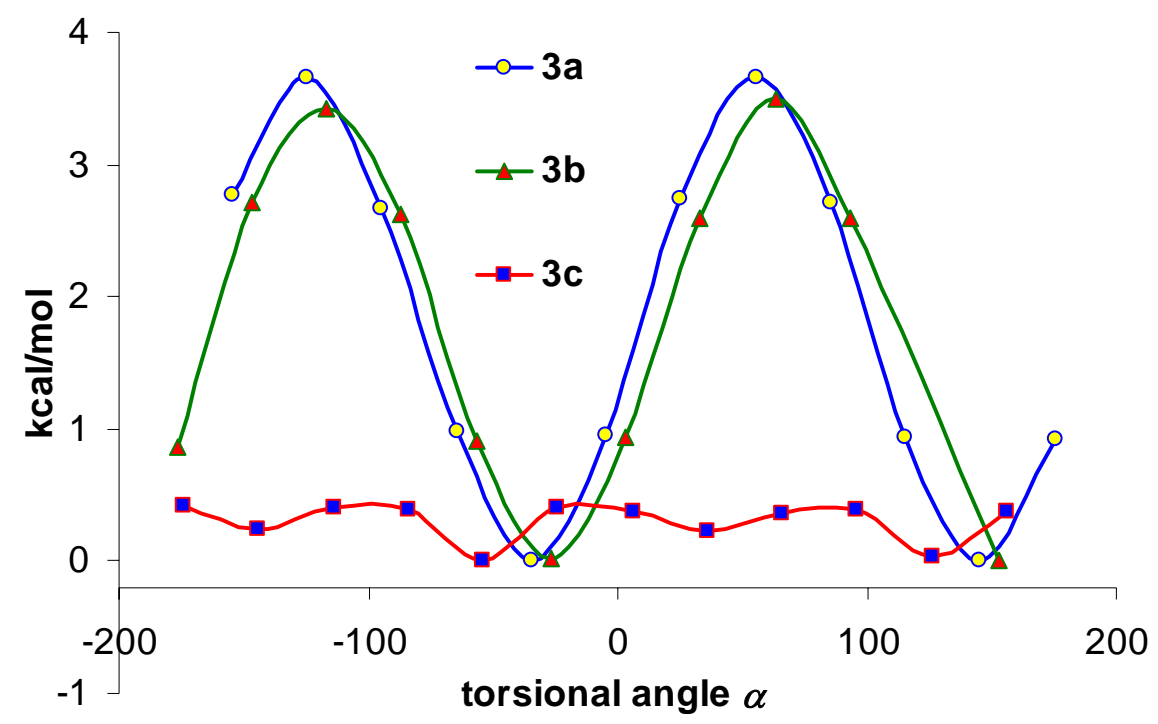

Figure 2. Calculated torsional potentials for compounds 3a-c.

After optimization, absolute ${ }^{1} \mathrm{H}$ and ${ }^{13} \mathrm{C}$ NMR chemical shifts $(\delta / \mathrm{ppm})$ were calculated with the GIAO method without solvent or in methanol at HF, B3LYP and MPW1PW91 levels using the $6-31+\mathrm{G}(\mathrm{d}, \mathrm{p})$ and $6-311++\mathrm{G}(2 \mathrm{~d}, \mathrm{p})$ basis sets. Relative chemical shifts were estimated by using the corresponding TMS shieldings calculated in advance at the same theoretical level as the reference.

\section{Results and Discussion}

\section{Synthesis of 4-cyclo(propyl- and butyl)-1-ethylpyridinium bromides (3a-c)}

Previously the alkylation of $\mathrm{C}$-H acids with dihalogenides was performed in two-phase system in $50 \%$ solution of sodium hydroxide in the presence of triethylbenzylammonium chloride as catalyst.Error! Bookmark not defined. Considerable yields of cycloalkanes from C-H acids also are obtained in system DMSO- $\mathrm{K}_{2} \mathrm{CO}_{3}{ }^{19}$

4-Methylpyridinium salt $\mathbf{1}$ also is $\mathrm{C}-\mathrm{H}$ acid due to the positive charge at the nitrogen atom. The synthesis of cylopropyl- and cyclobutylpyridinium salts 3 was performed by treating of 1ethyl-4-methylpyridinium bromide 1 with dibromides $\mathbf{2}$ (Scheme 1).
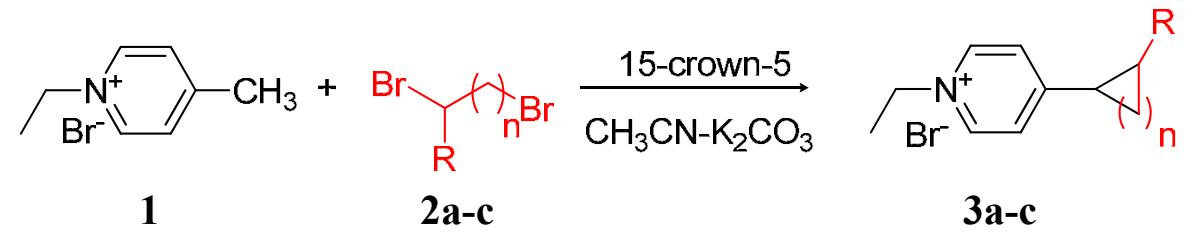

\begin{tabular}{l|l|l|l} 
& $\mathbf{a}$ & $\mathbf{b}$ & $\mathbf{c}$ \\
\hline $\mathrm{R}$ & $\mathrm{H}$ & $\mathrm{C}_{6} \mathrm{H}_{5}$ & $\mathrm{H}$ \\
\hline $\mathrm{n}$ & 1 & 1 & 2
\end{tabular}

Scheme 1 
A highly efficient synthesis was performed under PTC condition in solid-liquid anh. $\mathrm{K}_{2} \mathrm{CO}_{3}-$ anh. acetonitrile system. The influence of 15-crown-5 ether, dicyclohexyl-18-crown-6 ether, triethylbenzylammonium chloride (TEBA-Cl), ethyltriphenylphosphonium bromide as PT catalysts was tested by HPLC method. In all cases, the use of catalysts has accelerated reactions. Also we found, that in contrast with monoalkylation,Error! Bookmark not defined. this cyclization reaction required equimolar PT catalysts quantities. The catalytic activity order was found to be as follows: 15-crown-5 > dicyclohexyl-18-crown-6 > TEBA-Cl > ethyltriphenylphosphonium bromide. The cycloalkylpyridinium salts 3a-c was stored in a capped vials at $4{ }^{\circ} \mathrm{C}$ temperature, without noticeable decomposition over a period of several months.

\section{Calculation}

\section{${ }^{1}$ H-NMR spectra of (3a-c)}

Compound 3b, with phenyl subtituent in cyclopropan ring, can exist as cis and trans stereoisomers. In order to investigate obtained in our reaction compound structure, we have performed a theoretical study of compounds 3a-c (Figure 3).

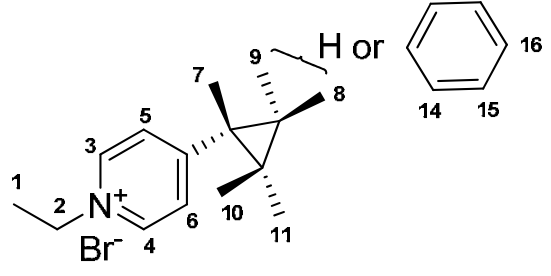

3a-b

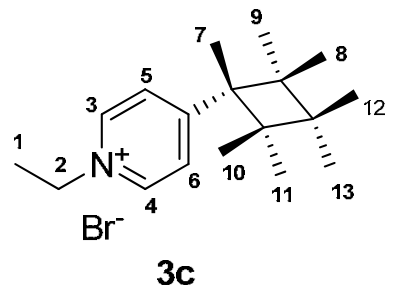

$3 \mathrm{c}$

Figure 3. Numbering of $\mathrm{H}$ atoms.

Table 1. Experimental and calculated ${ }^{1} \mathrm{H}-\mathrm{NMR}$ chemical shifts $(\delta / \mathrm{ppm})$ of compounds 3a-c

\begin{tabular}{|c|c|c|c|c|c|c|}
\hline \multirow{2}{*}{$\begin{array}{c}\text { Compound } \\
\text { No. }\end{array}$} & \multirow{2}{*}{$\begin{array}{c}\mathrm{H}- \\
\text { location }^{\mathrm{a}}\end{array}$} & \multirow{2}{*}{$\begin{array}{l}\text { Calc. } \\
\text { level }^{b}\end{array}$} & \multicolumn{3}{|c|}{ Method } & \multirow{2}{*}{$\operatorname{Exp}^{c}$} \\
\hline & & & $\mathrm{HF}$ & B3LYP & MPW1PW91 & \\
\hline \multirow[t]{9}{*}{$3 a$} & 1 & $\mathrm{a}$ & 1.61 & 1.59 & 1.58 & 1.63 \\
\hline & & $b$ & 1.67 & 1.67 & 1.65 & \\
\hline & & $\mathrm{c}$ & 1.55 & 1.56 & 1.54 & \\
\hline & 2 & $\mathrm{a}$ & 4.46 & 4.46 & 4.48 & 4.63 \\
\hline & & $\mathrm{b}$ & 4.23 & 4.35 & 4.35 & \\
\hline & & $\mathrm{c}$ & 4.43 & 4.52 & 4.53 & \\
\hline & 3,4 & $\mathrm{a}$ & 9.24 & 8.63 & 8.75 & 8.84 \\
\hline & & $\mathrm{b}$ & 8.58 & 8.16 & 8.22 & \\
\hline & & $\mathrm{c}$ & 9.19 & 8.72 & 8.80 & \\
\hline
\end{tabular}


Table 1. Continued

\begin{tabular}{|c|c|c|c|c|c|c|}
\hline \multirow{2}{*}{$\begin{array}{c}\text { Compound } \\
\text { No. }\end{array}$} & \multirow{2}{*}{$\begin{array}{c}\mathrm{H}- \\
\text { location }^{\mathrm{a}}\end{array}$} & \multirow{2}{*}{$\begin{array}{l}\text { Calc. } \\
\text { level }^{\mathrm{b}}\end{array}$} & \multicolumn{3}{|c|}{ Method } & \multirow[b]{2}{*}{ Exp. ${ }^{\circ}$} \\
\hline & & & HF & B3LYP & MPW1PW91 & \\
\hline & \multirow[t]{3}{*}{5,6} & $\mathrm{a}$ & 8.00 & 7.94 & 8.07 & \multirow[t]{3}{*}{7.80} \\
\hline & & b & 7.59 & 7.63 & 7.72 & \\
\hline & & c & 7.93 & 7.99 & 8.08 & \\
\hline & \multirow[t]{3}{*}{$8 \mathrm{a}$} & $\mathrm{a}$ & 2.21 & 2.38 & 2.44 & \multirow[t]{3}{*}{2.34} \\
\hline & & b & 1.80 & 2.10 & 2.11 & \\
\hline & & c & 2.23 & 2.46 & 2.48 & \\
\hline & \multirow[t]{3}{*}{$9 a, 10 a$} & $\mathrm{a}$ & 1.54 & 1.75 & 1.77 & \multirow[t]{3}{*}{1.48} \\
\hline & & b & 1.81 & 2.10 & 2.08 & \\
\hline & & $\mathrm{c}$ & 1.46 & 1.71 & 1.70 & \\
\hline & \multirow[t]{12}{*}{$9 b, 10 b$} & $\mathrm{a}$ & 1.21 & 1.27 & 1.31 & \multirow[t]{12}{*}{1.19} \\
\hline & & $\mathrm{b}$ & 1.19 & 1.38 & 1.39 & \\
\hline & & $\mathrm{c}$ & 1.15 & 1.28 & 1.30 & \\
\hline \multirow[t]{3}{*}{$|\Delta \delta|_{\mathrm{T}}$} & & $\mathrm{a}$ & 1.00 & 0.93 & 1.08 & \\
\hline & & b & 1.79 & 2.23 & 2.04 & \\
\hline & & $\mathrm{c}$ & 0.94 & 0.91 & 0.97 & \\
\hline \multirow[t]{3}{*}{$\mathrm{R}^{2}$} & & $\mathrm{a}$ & 0.9955 & 0.9999 & 0.9956 & \\
\hline & & $\mathrm{b}$ & 0.9924 & 0.9913 & 0.9912 & \\
\hline & & c & 0.9987 & 0.9980 & 0.9977 & \\
\hline \multirow[t]{3}{*}{ r.m.s. } & & $\mathrm{a}$ & 0.20 & 0.03 & 0.19 & \\
\hline & & $\mathrm{b}$ & 0.29 & 0.30 & 0.30 & \\
\hline & & $\mathrm{c}$ & 0.13 & 0.15 & 0.17 & \\
\hline \multirow[t]{14}{*}{$\mathbf{3 b}$ trans } & 1 & $\mathrm{a}$ & 1.63 & 1.57 & 1.56 & \multirow[t]{3}{*}{1.62} \\
\hline & & $\mathrm{b}$ & 1.66 & 1.66 & 1.64 & \\
\hline & & $\mathrm{c}$ & 1.55 & 1.53 & 1.51 & \\
\hline & \multirow[t]{3}{*}{2} & $\mathrm{a}$ & 4.48 & 4.46 & 4.49 & \multirow[t]{3}{*}{4.60} \\
\hline & & b & 4.21 & 4.29 & 4.30 & \\
\hline & & c & 4.44 & 4.49 & 4.51 & \\
\hline & \multirow[t]{3}{*}{3,4} & $\mathrm{a}$ & 9.28 & 8.64 & 8.77 & \multirow[t]{3}{*}{8.78} \\
\hline & & $\mathrm{b}$ & 8.55 & 8.08 & 8.16 & \\
\hline & & c & 9.21 & 8.72 & 8.80 & \\
\hline & \multirow[t]{3}{*}{5,6} & $\mathrm{a}$ & 8.06 & 7.97 & 8.11 & \multirow[t]{3}{*}{7.84} \\
\hline & & $\mathrm{b}$ & 7.57 & 7.55 & 7.64 & \\
\hline & & $\mathrm{c}$ & 7.97 & 8.05 & 8.14 & \\
\hline & \multirow{2}{*}{$8 a$} & $\mathrm{a}$ & 2.57 & 2.80 & 2.87 & \multirow[t]{2}{*}{2.68} \\
\hline & & $\mathrm{b}$ & 2.26 & 2.64 & 2.68 & \\
\hline
\end{tabular}


Table 1. Continued

\begin{tabular}{|c|c|c|c|c|c|c|}
\hline \multirow{2}{*}{$\begin{array}{c}\text { Compound } \\
\text { No. }\end{array}$} & \multirow{2}{*}{$\begin{array}{c}\mathrm{H}- \\
\text { location }^{\mathrm{a}}\end{array}$} & \multirow{2}{*}{$\begin{array}{l}\text { Calc. } \\
\text { level }^{\text {b }}\end{array}$} & \multicolumn{3}{|c|}{ Method } & \multirow[b]{2}{*}{ Exp. } \\
\hline & & & $\mathrm{HF}$ & B3LYP & MPW1PW91 & \\
\hline & & $\mathrm{c}$ & 2.57 & 2.83 & 2.88 & \\
\hline & $9 b$ & $\mathrm{a}$ & 2.28 & 2.46 & 2.55 & 2.54 \\
\hline & & $\mathrm{b}$ & 1.76 & 2.10 & 2.14 & \\
\hline & & $\mathrm{c}$ & 2.27 & 2.56 & 2.61 & \\
\hline & $10 \mathrm{a}$ & $\mathrm{a}$ & 2.04 & 2.23 & 2.28 & 1.89 \\
\hline & & b & 1.97 & 2.22 & 2.23 & \\
\hline & & $\mathrm{c}$ & 1.92 & 2.11 & 2.13 & \\
\hline & $10 \mathrm{~b}$ & $\mathrm{a}$ & 2.25 & 2.43 & 2.48 & 1.96 \\
\hline & & $\mathrm{b}$ & 2.57 & 2.74 & 2.74 & \\
\hline & & $\mathrm{c}$ & 2.14 & 2.37 & 2.38 & \\
\hline & 13 & $\mathrm{a}$ & 7.90 & 7.60 & 7.72 & 7.29 \\
\hline & & b & 7.44 & 7.39 & 7.47 & \\
\hline & & $\mathrm{c}$ & 7.85 & 7.73 & 7.83 & \\
\hline & 14,15 & $\mathrm{a}$ & 8.07 & 7.79 & 7.93 & 7.21 \\
\hline & & $\mathrm{b}$ & 7.92 & 7.82 & 7.91 & \\
\hline & & $\mathrm{c}$ & 8.03 & 7.84 & 7.94 & \\
\hline \multirow[t]{3}{*}{$|\Delta \delta|_{\mathrm{T}}$} & & $\mathrm{a}$ & 3.12 & 2.37 & 2.68 & \\
\hline & & b & 5.11 & 4.12 & 4.17 & \\
\hline & & $\mathrm{c}$ & 2.75 & 2.36 & 2.73 & \\
\hline \multirow[t]{3}{*}{$\mathrm{R}^{2}$} & & $\mathrm{a}$ & 0.9927 & 0.9917 & 0.9912 & \\
\hline & & b & 0.9749 & 0.9740 & 0.9744 & \\
\hline & & $\mathrm{c}$ & 0.9934 & 0.9930 & 0.9924 & \\
\hline \multirow[t]{3}{*}{ r.m.s. } & & $\mathrm{a}$ & 0.28 & 0.28 & 0.29 & \\
\hline & & $\mathrm{b}$ & 0.49 & 0.47 & 0.47 & \\
\hline & & $\mathrm{c}$ & 0.27 & 0.26 & 0.27 & \\
\hline \multirow[t]{10}{*}{$\mathbf{3 b}$ cis } & 1 & $\mathrm{a}$ & 1.52 & 1.51 & 1.50 & 1.62 \\
\hline & & b & 1.56 & 1.55 & 1.53 & \\
\hline & & $\mathrm{c}$ & 1.45 & 1.48 & 1.46 & \\
\hline & 2 & $\mathrm{a}$ & 4.27 & 4.33 & 4.34 & 4.60 \\
\hline & & $\mathrm{b}$ & 4.05 & 4.14 & 4.14 & \\
\hline & & $\mathrm{c}$ & 4.24 & 4.32 & 4.32 & \\
\hline & 3,4 & $\mathrm{a}$ & 8.97 & 8.28 & 8.39 & 8.78 \\
\hline & & b & 8.27 & 7.87 & 7.93 & \\
\hline & & c & 8.94 & 8.52 & 8.61 & \\
\hline & 5,6 & $\mathrm{a}$ & 7.78 & 7.72 & 7.84 & 7.84 \\
\hline
\end{tabular}


Table 1. Continued

\begin{tabular}{|c|c|c|c|c|c|c|}
\hline \multirow{2}{*}{$\begin{array}{c}\text { Compound } \\
\text { No. }\end{array}$} & \multirow{2}{*}{$\begin{array}{c}\mathrm{H}- \\
\text { location }^{\mathrm{a}}\end{array}$} & \multirow{2}{*}{$\begin{array}{l}\text { Calc. } \\
\text { level }^{\mathrm{b}}\end{array}$} & \multicolumn{3}{|c|}{ Method } & \multirow[b]{2}{*}{ Exp. ${ }^{\circ}$} \\
\hline & & & HF & B3LYP & MPW1PW91 & \\
\hline & & b & 7.29 & 7.37 & 7.45 & \\
\hline & & $\mathrm{c}$ & 7.70 & 7.79 & 7.87 & \\
\hline & $8 a$ & $\mathrm{a}$ & 2.74 & 3.12 & 3.18 & 2.68 \\
\hline & & b & 2.13 & 2.58 & 2.60 & \\
\hline & & $\mathrm{c}$ & 2.72 & 3.12 & 3.14 & \\
\hline & $9 \mathrm{a}$ & $\mathrm{a}$ & 3.28 & 3.76 & 3.77 & 2.54 \\
\hline & & $\mathrm{b}$ & 3.30 & 3.81 & 3.80 & \\
\hline & & $\mathrm{c}$ & 3.22 & 3.73 & 3.73 & \\
\hline & $10 \mathrm{a}$ & $\mathrm{a}$ & 2.14 & 2.21 & 2.28 & 1.89 \\
\hline & & b & 1.77 & 1.96 & 1.99 & \\
\hline & & $\mathrm{c}$ & 2.07 & 2.24 & 2.27 & \\
\hline & $10 \mathrm{~b}$ & $\mathrm{a}$ & 1.93 & 2.12 & 2.15 & 1.96 \\
\hline & & b & 2.25 & 2.48 & 2.46 & \\
\hline & & $\mathrm{c}$ & 1.83 & 2.00 & 2.00 & \\
\hline & 13 & $\mathrm{a}$ & 8.03 & 7.70 & 7.83 & 7.29 \\
\hline & & $\mathrm{b}$ & 7.66 & 7.55 & 7.62 & \\
\hline & & $\mathrm{c}$ & 7.97 & 7.83 & 7.91 & \\
\hline & 14,15 & $\mathrm{a}$ & 7.96 & 7.74 & 7.86 & 7.21 \\
\hline & & $\mathrm{b}$ & 7.90 & 7.80 & 7.88 & \\
\hline & & $\mathrm{c}$ & 7.91 & 7.76 & 7.86 & \\
\hline$|\Delta \delta|_{\mathrm{T}}$ & & $\mathrm{a}$ & 3.26 & 4.09 & 4.27 & \\
\hline & & $\mathrm{b}$ & 5.02 & 4.57 & 4.75 & \\
\hline & & $\mathrm{c}$ & 3.26 & 3.85 & 3.99 & \\
\hline $\mathrm{R}^{2}$ & & $\mathrm{a}$ & 0.9855 & 0.9738 & 0.9731 & \\
\hline & & $\mathrm{b}$ & 0.9709 & 0.9592 & 0.9596 & \\
\hline & & $\mathrm{c}$ & 0.9855 & 0.9760 & 0.9756 & \\
\hline r.m.s. & & $\mathrm{a}$ & 0.38 & 0.47 & 0.48 & \\
\hline & & b & 0.51 & 0.57 & 0.57 & \\
\hline & & c & 0.38 & 0.46 & 0.47 & \\
\hline $3 c$ & 1 & $\mathrm{a}$ & 1.62 & 1.60 & 1.58 & 1.64 \\
\hline & & $\mathrm{b}$ & 1.70 & 1.70 & 1.68 & \\
\hline & & $\mathrm{c}$ & 1.55 & 1.55 & 1.55 & \\
\hline & 2 & $\mathrm{a}$ & 4.51 & 4.51 & 4.52 & 4.65 \\
\hline & & b & 4.30 & 4.42 & 4.42 & \\
\hline & & c & 4.47 & 4.56 & 4.58 & \\
\hline
\end{tabular}


Table 1. Continued

\begin{tabular}{|c|c|c|c|c|c|c|}
\hline \multirow{2}{*}{$\begin{array}{c}\text { Compound } \\
\text { No. }\end{array}$} & \multirow{2}{*}{$\begin{array}{c}\mathrm{H}- \\
\text { location }^{\mathrm{a}}\end{array}$} & \multirow{2}{*}{$\begin{array}{l}\text { Calc. } \\
\text { level }\end{array}$} & \multicolumn{3}{|c|}{ Method } & \multirow{2}{*}{ Exp. $^{\mathrm{c}}$} \\
\hline & & & $\mathrm{HF}$ & B3LYP & MPW1PW91 & \\
\hline & \multirow[t]{3}{*}{3,4} & $\mathrm{a}$ & 9.24 & 8.66 & 8.77 & \multirow[t]{3}{*}{8.90} \\
\hline & & b & 8.72 & 8.32 & 8.38 & \\
\hline & & $\mathrm{c}$ & 9.18 & 8.74 & 8.93 & \\
\hline & \multirow[t]{3}{*}{5,6} & $\mathrm{a}$ & 8.35 & 8.31 & 8.44 & \multirow[t]{3}{*}{7.95} \\
\hline & & $\mathrm{b}$ & 8.06 & 8.12 & 8.20 & \\
\hline & & $\mathrm{c}$ & 8.26 & 8.33 & 8.50 & \\
\hline & \multirow[t]{3}{*}{$8 \mathrm{a}$} & $\mathrm{a}$ & 3.75 & 3.89 & 3.93 & \multirow[t]{3}{*}{3.91} \\
\hline & & $\mathrm{b}$ & 3.45 & 3.72 & 3.74 & \\
\hline & & $\mathrm{c}$ & 3.77 & 4.01 & 4.06 & \\
\hline & \multirow[t]{3}{*}{$9 a, 10 a$} & $\mathrm{a}$ & 2.31 & 2.51 & 2.47 & \multirow[t]{3}{*}{2.51} \\
\hline & & $\mathrm{b}$ & 2.49 & 2.72 & 2.67 & \\
\hline & & $\mathrm{c}$ & 2.26 & 2.48 & 2.46 & \\
\hline & \multirow[t]{3}{*}{$9 b, 10 b$} & $\mathrm{a}$ & 2.40 & 2.46 & 2.48 & \multirow[t]{3}{*}{2.31} \\
\hline & & $\mathrm{b}$ & 2.17 & 2.29 & 2.30 & \\
\hline & & $\mathrm{c}$ & 2.30 & 2.40 & 2.41 & \\
\hline & \multirow[t]{3}{*}{$11 \mathrm{a}$} & $\mathrm{a}$ & 2.18 & 2.23 & 2.23 & \multirow[t]{3}{*}{2.17} \\
\hline & & b & 2.37 & 2.44 & 2.42 & \\
\hline & & c & 2.10 & 2.20 & 2.21 & \\
\hline & \multirow[t]{6}{*}{$11 \mathrm{~b}$} & $\mathrm{a}$ & 1.92 & 2.03 & 2.00 & \multirow[t]{6}{*}{1.95} \\
\hline & & $\mathrm{b}$ & 2.15 & 2.22 & 2.19 & \\
\hline & & $\mathrm{c}$ & 1.88 & 1.98 & 2.00 & \\
\hline \multirow[t]{3}{*}{$|\Delta \delta|_{\mathrm{T}}$} & & $\mathrm{a}$ & 1.37 & 1.07 & 1.15 & \\
\hline & & $\mathrm{b}$ & 1.71 & 2.01 & 1.88 & \\
\hline & & $\mathrm{c}$ & 1.42 & 1.00 & 1.13 & \\
\hline \multirow[t]{3}{*}{$\mathrm{R}^{2}$} & & $\mathrm{a}$ & 0.9975 & 0.9959 & 0.9954 & \\
\hline & & b & 0.9926 & 0.9921 & 0.9922 & \\
\hline & & c & 0.9984 & 0.9968 & 0.9966 & \\
\hline \multirow[t]{3}{*}{ r.m.s. } & & $\mathrm{a}$ & 0.15 & 0.18 & 0.20 & \\
\hline & & b & 0.24 & 0.24 & 0.24 & \\
\hline & & c & 0.12 & 0.17 & 0.18 & \\
\hline
\end{tabular}

\footnotetext{
${ }^{\mathrm{a}}$ Mean values for $\mathrm{CH}_{2}$ and $\mathrm{CH}_{3}$ group protons.

b. $\mathbf{a}-6-31+\mathrm{G}(\mathrm{d}, \mathrm{p})$ in methanol; b - 6-311++G(2d,p); $\mathbf{c}-6-311++\mathrm{G}(2 \mathrm{~d}, \mathrm{p})$ in methanol.

${ }^{c}$ Centre of multiplet.

a $-6-31+\mathrm{G}(\mathrm{d}, \mathrm{p})$ in methanol; b - 6-311++G(2d,p); $\mathbf{c}-6-311++\mathrm{G}(2 \mathrm{~d}, \mathrm{p})$ in methanol.

${ }^{a}$ Mean values for $\mathrm{CH}_{2}$ and $\mathrm{CH}_{3}$ group protons.

${ }^{b}$ Centre of multiplet.
} 
The assigned proton chemical shifts were supported by the GIAO method in gas phase or in methanol by using the IEF-PCM method calculated values. Comparisons between experimental and theoretical chemical shift data were made using the following statistical parameters: total absolute error between computed and experimental chemical shifts $\left(|\Delta \delta|_{\mathrm{T}}, \mathrm{ppm}\right)^{20}$ and correlation coefficients $\left(\mathrm{R}^{2}\right)$ deriving from linear regression of computed chemical shifts to experimental values. Calculated root-mean-squared error (r.m.s.) values were also used to compare the per atom performance of the various methods employed. In all cases, an excellent correlation was observed between experimental and calculated ${ }^{1} \mathrm{H}-\mathrm{NMR}$ values (Table 1). Using of the solvent effect in calculation gives a more accurate result than in gas phase calculation. Computed chemical shifts are slightly better with lager basis set $6-311++G(2 d, p)$ as compared with 6$31+\mathrm{G}(\mathrm{d}, \mathrm{p})$. More satisfactory method was B3LYP/6-311++G(2d,p) in methanol. The molecular modeling data clearly indicate the formation of trans- isomer of compound $\mathbf{3 b}$ (Table 1 highlights). As can be seen from Table 1 that the largest contributions to the ${ }^{1} \mathrm{H}$ shift error in $\mathbf{3 b}$ cis-stereoisomer are associated with the H-9a and H-8a atoms: $1.05 \mathrm{ppm}$ (39\%) of the error is due to H-9a and $0.58 \mathrm{ppm}(23 \%)$ to $\mathrm{H}-8 \mathrm{a}$. The $\mathbf{3 b}$ trans- stereoisomer ${ }^{1} \mathrm{H}$ shift error on the $\mathrm{H}-9 \mathrm{~b}$ was only $0.15 \mathrm{ppm}(6 \%)$ and $\mathrm{H}-8 \mathrm{a}-0.02 \mathrm{ppm}(1 \%)$.

\section{${ }^{13}$ C NMR spectra of (3a-c)}

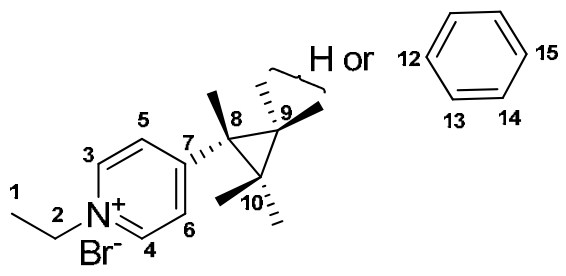

3a-b

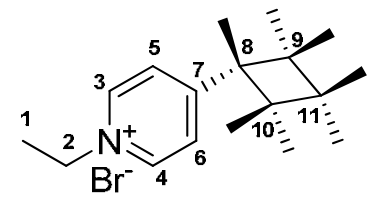

3c

Figure 4. Numbering of $\mathrm{C}$ atoms.

Table 2. Experimental and calculated ${ }^{13} \mathrm{C}-\mathrm{NMR}$ chemical shifts $(\delta / \mathrm{ppm})$ of compounds 3a-c

\begin{tabular}{|c|c|c|c|c|c|c|}
\hline \multirow[b]{2}{*}{$\begin{array}{c}\text { Compound } \\
\text { No. }\end{array}$} & \multirow[b]{2}{*}{ C-location } & \multicolumn{4}{|c|}{ Method } & \multirow[b]{2}{*}{ Exp. } \\
\hline & & $\begin{array}{l}\text { Calc. } \\
\text { level } \\
\underset{a}{a}\end{array}$ & $\mathrm{HF}$ & B3LYP & MPW1PW91 & \\
\hline \multirow[t]{7}{*}{$3 a$} & 1 & $\mathrm{a}$ & 18.96 & 19.92 & 19.47 & 15.26 \\
\hline & & $\mathrm{b}$ & 18.93 & 20.01 & 19.37 & \\
\hline & & $\mathrm{c}$ & 18.80 & 19.67 & 19.07 & \\
\hline & 2 & $\mathrm{a}$ & 53.07 & 59.86 & 59.16 & 57.08 \\
\hline & & $\mathrm{b}$ & 55.69 & 63.05 & 61.74 & \\
\hline & & $\mathrm{c}$ & 54.40 & 61.56 & 60.29 & \\
\hline & 3.4 & $\mathrm{a}$ & 150.20 & 139.18 & 140.56 & 144.28 \\
\hline
\end{tabular}


Method

Compound C- Calc.

No. location level

HF B3LYP MPW1PW91 Exp.

\begin{tabular}{|c|c|c|c|c|c|c|}
\hline & \multirow{5}{*}{5,6} & $b$ & 154.38 & 146.25 & 145.87 & \multirow{5}{*}{125.36} \\
\hline & & $\mathrm{c}$ & 156.68 & 148.49 & 148.13 & \\
\hline & & $\mathrm{a}$ & 119.00 & 121.75 & 122.66 & \\
\hline & & $\mathrm{b}$ & 124.33 & 129.61 & 129.04 & \\
\hline & & $\mathrm{c}$ & 124.25 & 130.03 & 129.44 & \\
\hline & \multirow[t]{3}{*}{7} & $\mathrm{a}$ & 179.54 & 167.21 & 167.65 & \multirow[t]{3}{*}{168.16} \\
\hline & & $\mathrm{b}$ & 190.17 & 181.29 & 179.71 & \\
\hline & & $\mathrm{c}$ & 186.03 & 177.75 & 176.17 & \\
\hline & \multirow[t]{3}{*}{8} & $\mathrm{a}$ & 12.26 & 22.52 & 21.98 & \multirow[t]{3}{*}{17.51} \\
\hline & & $\mathrm{b}$ & 13.44 & 24.57 & 23.97 & \\
\hline & & $\mathrm{c}$ & 12.37 & 22.29 & 21.79 & \\
\hline & \multirow[t]{12}{*}{9,10} & $\mathrm{a}$ & 13.51 & 20.87 & 20.77 & \multirow[t]{12}{*}{16.79} \\
\hline & & $\mathrm{b}$ & 16.82 & 26.51 & 25.74 & \\
\hline & & $\mathrm{c}$ & 13.24 & 21.68 & 21.11 & \\
\hline \multirow[t]{3}{*}{$|\Delta \delta|_{\mathrm{T}}$} & & $\mathrm{a}$ & 39.89 & 26.20 & 21.67 & \\
\hline & & $\mathrm{b}$ & 42.32 & 46.84 & 41.00 & \\
\hline & & $\mathrm{c}$ & 46.29 & 37.03 & 31.55 & \\
\hline \multirow[t]{3}{*}{$\mathrm{R} 2$} & & $\mathrm{a}$ & 0.9937 & 0.9992 & 0.9995 & \\
\hline & & $\mathrm{b}$ & 0.9929 & 0.9969 & 0.9974 & \\
\hline & & $\mathrm{c}$ & 0.9943 & 0.9994 & 0.9996 & \\
\hline \multirow[t]{3}{*}{ r.m.s. } & & $\mathrm{a}$ & 6.15 & 2.00 & 1.59 & \\
\hline & & $\mathrm{b}$ & 6.80 & 4.08 & 3.73 & \\
\hline & & $\mathrm{c}$ & 6.06 & 1.76 & 1.45 & \\
\hline \multirow[t]{11}{*}{$\mathbf{3 b}$ trans } & \multirow[t]{3}{*}{1} & $\mathrm{a}$ & 18.99 & 19.87 & 19.47 & \multirow[t]{3}{*}{16.70} \\
\hline & & b & 18.90 & 19.80 & 19.19 & \\
\hline & & $\mathrm{c}$ & 19.19 & 20.14 & 19.52 & \\
\hline & \multirow[t]{3}{*}{2} & $\mathrm{a}$ & 53.09 & 59.51 & 58.79 & \multirow[t]{3}{*}{57.23} \\
\hline & & $\mathrm{b}$ & 55.25 & 62.37 & 61.06 & \\
\hline & & $\mathrm{c}$ & 54.82 & 61.94 & 60.66 & \\
\hline & \multirow[t]{3}{*}{3,4} & $\mathrm{a}$ & 150.29 & 138.90 & 140.36 & \multirow[t]{3}{*}{144.41} \\
\hline & & $\mathrm{b}$ & 154.26 & 145.88 & 145.59 & \\
\hline & & $\mathrm{c}$ & 157.17 & 148.83 & 148.50 & \\
\hline & \multirow[t]{2}{*}{5,6} & $\mathrm{a}$ & 119.13 & 121.93 & 122.94 & \multirow[t]{2}{*}{125.49} \\
\hline & & $\mathrm{b}$ & 123.74 & 129.24 & 128.72 & \\
\hline
\end{tabular}




\begin{tabular}{|c|c|c|c|c|c|c|}
\hline & \multirow{4}{*}{7} & $\mathrm{c}$ & 124.78 & 130.47 & 129.77 & \multirow{4}{*}{165.90} \\
\hline & & $\mathrm{a}$ & 177.08 & 164.03 & 164.46 & \\
\hline & & $\mathrm{b}$ & 188.15 & 176.74 & 175.57 & \\
\hline & & $\mathrm{c}$ & 183.79 & 174.67 & 173.13 & \\
\hline & \multirow[t]{3}{*}{8} & $\mathrm{a}$ & 25.98 & 37.61 & 36.61 & \multirow[t]{3}{*}{29.39} \\
\hline & & $\mathrm{b}$ & 28.05 & 41.93 & 40.39 & \\
\hline & & $\mathrm{c}$ & 26.88 & 38.66 & 37.25 & \\
\hline & \multirow[t]{3}{*}{9} & $\mathrm{a}$ & 30.38 & 43.51 & 42.60 & \multirow[t]{3}{*}{34.18} \\
\hline & & $\mathrm{b}$ & 38.55 & 54.41 & 52.48 & \\
\hline & & $\mathrm{c}$ & 31.09 & 44.76 & 43.17 & \\
\hline & \multirow[t]{3}{*}{10} & $\mathrm{a}$ & 19.74 & 26.67 & 26.34 & \multirow[t]{3}{*}{22.65} \\
\hline & & $\mathrm{b}$ & 21.11 & 28.95 & 28.05 & \\
\hline & & $\mathrm{c}$ & 19.87 & 27.79 & 26.84 & \\
\hline & \multirow[t]{3}{*}{12} & $\mathrm{a}$ & 143.03 & 140.05 & 139.79 & \multirow[t]{3}{*}{141.20} \\
\hline & & $\mathrm{b}$ & 141.01 & 142.01 & 140.01 & \\
\hline & & $\mathrm{c}$ & 148.65 & 149.54 & 147.69 & \\
\hline & \multirow[t]{3}{*}{13} & $\mathrm{a}$ & 126.57 & 124.00 & 125.37 & \multirow[t]{3}{*}{127.10} \\
\hline & & $\mathrm{b}$ & 131.03 & 130.21 & 129.78 & \\
\hline & & $\mathrm{c}$ & 132.55 & 131.29 & 130.89 & \\
\hline & \multirow[t]{3}{*}{14} & $\mathrm{a}$ & 130.30 & 124.96 & 125.71 & \multirow[t]{3}{*}{129.66} \\
\hline & & $\mathrm{b}$ & 137.15 & 135.79 & 135.20 & \\
\hline & & $\mathrm{c}$ & 136.68 & 135.83 & 135.28 & \\
\hline & \multirow[t]{6}{*}{15} & $\mathrm{a}$ & 127.25 & 124.29 & 125.73 & \multirow[t]{12}{*}{127.83} \\
\hline & & $\mathrm{b}$ & 136.88 & 136.85 & 136.25 & \\
\hline & & $\mathrm{c}$ & 133.50 & 133.46 & 133.02 & \\
\hline \multirow[t]{3}{*}{$|\Delta \delta|_{\mathrm{T}}$} & & $\mathrm{a}$ & 43.55 & 50.46 & 40.89 & \\
\hline & & $\mathrm{b}$ & 65.95 & 82.42 & 72.94 & \\
\hline & & $\mathrm{c}$ & 70.22 & 75.62 & 63.99 & \\
\hline \multirow[t]{3}{*}{$\mathrm{R}^{2}$} & & $\mathrm{a}$ & 0.9951 & 0.9976 & 0.9983 & \\
\hline & & $\mathrm{b}$ & 0.9912 & 0.9909 & 0.9917 & \\
\hline & & $\mathrm{c}$ & 0.9958 & 0.9983 & 0.9988 & \\
\hline \multirow[t]{3}{*}{ r.m.s. } & & $\mathrm{a}$ & 4.33 & 2.66 & 2.24 & \\
\hline & & $\mathrm{b}$ & 5.98 & 5.44 & 5.20 & \\
\hline & & $\mathrm{c}$ & 4.19 & 2.44 & 2.03 & \\
\hline \multirow[t]{4}{*}{$\mathbf{3 b} c i s$} & \multirow[t]{3}{*}{1} & $\mathrm{a}$ & 18.88 & 19.57 & 19.04 & \multirow[t]{3}{*}{16.70} \\
\hline & & $\mathrm{b}$ & 18.86 & 19.78 & 19.20 & \\
\hline & & $\mathrm{c}$ & 19.14 & 20.09 & 19.51 & \\
\hline & 2 & $\mathrm{a}$ & 52.82 & 59.47 & 58.80 & 57.23 \\
\hline
\end{tabular}




\begin{tabular}{|c|c|c|c|c|c|c|}
\hline & & $\mathrm{b}$ & 55.10 & 62.14 & 60.86 & \\
\hline & & $\mathrm{c}$ & 54.56 & 61.61 & 60.32 & \\
\hline & 3,4 & $\mathrm{a}$ & 149.00 & 138.64 & 139.98 & 144.41 \\
\hline & & $\mathrm{b}$ & 152.79 & 144.63 & 144.30 & \\
\hline & & $\mathrm{c}$ & 156.05 & 148.10 & 147.72 & \\
\hline & 5,6 & $\mathrm{a}$ & 120.98 & 123.03 & 123.86 & 125.49 \\
\hline & & $\mathrm{b}$ & 126.15 & 131.18 & 130.53 & \\
\hline & & $\mathrm{c}$ & 126.62 & 133.22 & 132.59 & \\
\hline & 7 & $\mathrm{a}$ & 174.46 & 163.05 & 163.70 & 165.90 \\
\hline & & $\mathrm{b}$ & 186.18 & 176.53 & 175.06 & \\
\hline & & $\mathrm{c}$ & 181.42 & 172.96 & 171.57 & \\
\hline & 8 & $\mathrm{a}$ & 19.96 & 31.13 & 30.24 & 29.39 \\
\hline & & $\mathrm{b}$ & 20.00 & 33.03 & 32.04 & \\
\hline & & $\mathrm{c}$ & 20.32 & 32.91 & 31.93 & \\
\hline & 9 & $\mathrm{a}$ & 27.66 & 41.59 & 40.72 & 34.18 \\
\hline & & $\mathrm{b}$ & 31.58 & 46.02 & 44.71 & \\
\hline & & $\mathrm{c}$ & 28.12 & 41.83 & 40.63 & \\
\hline & 10 & $\mathrm{a}$ & 12.75 & 18.50 & 18.28 & 22.65 \\
\hline & & $\mathrm{b}$ & 19.47 & 28.35 & 27.56 & \\
\hline & & $\mathrm{c}$ & 12.46 & 19.01 & 18.51 & \\
\hline & 12 & $\mathrm{a}$ & 137.38 & 134.08 & 134.14 & 141.20 \\
\hline & & b & 138.50 & 137.98 & 136.39 & \\
\hline & & $\mathrm{c}$ & 143.01 & 142.83 & 141.26 & \\
\hline & 13 & $\mathrm{a}$ & 131.77 & 128.17 & 129.22 & 127.10 \\
\hline & & $\mathrm{b}$ & 136.71 & 135.67 & 135.17 & \\
\hline & & $\mathrm{c}$ & 138.05 & 137.10 & 136.56 & \\
\hline & 14 & $\mathrm{a}$ & 129.32 & 124.97 & 125.77 & 129.66 \\
\hline & & $\mathrm{b}$ & 137.02 & 136.51 & 135.93 & \\
\hline & & $\mathrm{c}$ & 135.94 & 135.23 & 134.69 & \\
\hline & 15 & $\mathrm{a}$ & 127.97 & 124.07 & 125.34 & 127.83 \\
\hline & & $b$ & 136.78 & 136.63 & 136.15 & \\
\hline & & $\mathrm{c}$ & 134.39 & 133.91 & 133.53 & \\
\hline$|\Delta \delta|_{\mathrm{T}}$ & & $\mathrm{a}$ & 59.08 & 46.13 & 39.50 & \\
\hline & & $\mathrm{b}$ & 77.40 & 73.14 & 65.98 & \\
\hline & & $\mathrm{c}$ & 84.33 & 64.34 & 55.36 & \\
\hline $\mathrm{R}^{2}$ & & $\mathrm{a}$ & 0.9946 & 0.9963 & 0.9965 & \\
\hline & & $b$ & 0.9919 & 0.9942 & 0.9941 & \\
\hline & & $\mathrm{c}$ & 0.9946 & 0.9969 & 0.9969 & \\
\hline
\end{tabular}




\begin{tabular}{|c|c|c|c|c|c|c|}
\hline \multirow[t]{3}{*}{ r.m.s. } & & $\mathrm{a}$ & 4.63 & 3.41 & 3.34 & \\
\hline & & b & 5.86 & 4.49 & 4.52 & \\
\hline & & $\mathrm{c}$ & 4.85 & 3.35 & 3.38 & \\
\hline \multirow[t]{24}{*}{$3 c$} & 1 & $\mathrm{a}$ & 18.61 & 19.64 & 19.19 & 16.48 \\
\hline & & $b$ & 19.04 & 20.21 & 19.56 & \\
\hline & & $\mathrm{c}$ & 18.85 & 19.79 & 19.64 & \\
\hline & 2 & $\mathrm{a}$ & 53.59 & 60.35 & 59.69 & 57.21 \\
\hline & & $b$ & 56.26 & 63.67 & 62.35 & \\
\hline & & $\mathrm{c}$ & 55.34 & 62.59 & 61.31 & \\
\hline & 3,4 & $\mathrm{a}$ & 150.20 & 139.60 & 140.99 & 144.64 \\
\hline & & $b$ & 154.64 & 146.86 & 146.49 & \\
\hline & & $\mathrm{c}$ & 157.14 & 149.38 & 149.29 & \\
\hline & 5,6 & $\mathrm{a}$ & 122.42 & 125.20 & 126.08 & 126.87 \\
\hline & & $\mathrm{b}$ & 128.41 & 133.65 & 133.06 & \\
\hline & & $\mathrm{c}$ & 128.22 & 133.99 & 133.47 & \\
\hline & 7 & $\mathrm{a}$ & 177.52 & 166.04 & 166.55 & 167.17 \\
\hline & & $\mathrm{b}$ & 187.66 & 180.30 & 178.80 & \\
\hline & & $\mathrm{c}$ & 184.57 & 177.08 & 174.88 & \\
\hline & 8 & $\mathrm{a}$ & 36.69 & 45.90 & 44.01 & 40.71 \\
\hline & & $\mathrm{b}$ & 37.91 & 47.86 & 45.81 & \\
\hline & & $\mathrm{c}$ & 37.41 & 46.66 & 44.97 & \\
\hline & 9,10 & $\mathrm{a}$ & 29.41 & 35.12 & 33.95 & 29.55 \\
\hline & & b & 30.20 & 36.55 & 35.06 & \\
\hline & & $\mathrm{c}$ & 29.59 & 35.32 & 33.89 & \\
\hline & 11 & $\mathrm{a}$ & 17.49 & 21.13 & 20.28 & 18.75 \\
\hline & & b & 17.85 & 20.55 & 19.62 & \\
\hline & & $\mathrm{c}$ & 17.70 & 20.57 & 20.06 & \\
\hline \multirow[t]{3}{*}{$|\Delta \delta|_{\mathrm{T}}$} & & $\mathrm{a}$ & 31.53 & 27.29 & 19.47 & \\
\hline & & $\mathrm{b}$ & 39.88 & 48.26 & 39.37 & \\
\hline & & $\mathrm{c}$ & 39.88 & 43.99 & 36.13 & \\
\hline \multirow[t]{3}{*}{$\mathrm{R}^{2}$} & & $\mathrm{a}$ & 0.9955 & 0.9989 & 0.9994 & \\
\hline & & $\mathrm{b}$ & 0.9951 & 0.9974 & 0.9981 & \\
\hline & & $\mathrm{c}$ & 0.9959 & 0.9993 & 0.9997 & \\
\hline \multirow[t]{3}{*}{ r.m.s. } & & $\mathrm{a}$ & 4.65 & 2.10 & 1.56 & \\
\hline & & b & 5.08 & 3.46 & 2.99 & \\
\hline & & $\mathrm{c}$ & 4.63 & 1.82 & 1.19 & \\
\hline
\end{tabular}


Isotopic ${ }^{13} \mathrm{C}$ values (computed with respect to TMS) for the B3LYP/6-311++G(2d,p) optimized geometries in gas-phase and methanol of compound 3a-c are presented in Table 2, together with the available experimental data. Comparison between experimental and theoretical chemical shift data was made by using the same as in ${ }^{1} \mathrm{H}$ chemical shift case criterions. As can be seen, the IEF-PCM method provides the excellent agreement of the 6- $311+\mathrm{G}(2 \mathrm{~d}, \mathrm{p})$ and 6$311+\mathrm{G}(2 \mathrm{~d}, \mathrm{p})$.values with the experiment, far better than in the case of gas-phase approaches. Total absolute error $\mid \Delta \delta_{T}$ values were smallest with the smaller basis set $631+\mathrm{G}(\mathrm{d}, \mathrm{p})$, but other two criterions $R^{2}$ and r.m.s. were better with the bigger basis set $6-311+G(2 d, p)$. More precise calculated ${ }^{13} \mathrm{C}$ chemical shift values are given by the IEF-PCM/MPW1PW91/6- $311+\mathrm{G}(2 \mathrm{~d}, \mathrm{p})$ level of theory. Analysis of the calculated ${ }^{13} \mathrm{C}$ data of the stereoisomers 3b-cis and 3b-trans show very close values and did not exceed the computational errors. In agreement with previous study, ${ }^{20,21}$ we obtained better results for the stereochemical distinction of studied compounds by using the ${ }^{1} \mathrm{H}$ calculated values as compared with the ${ }^{13} \mathrm{C}$ ones.

In conclusion, we have demonstrated a simple one-pot synthesis of 4-cyclopropyl- and 4cyclobutylpyridinium salts from easily available $\alpha, \omega$-dibromoalkanes and 4-methylpyridinium salt. The experimental values of ${ }^{1} \mathrm{H}$ and ${ }^{13} \mathrm{C}$ chemical shifts were found to be in a good agreement with the values obtained from IEF-PCM/GIAO/DFT calculations. More precise calculated values are given by the IEF-PCM/B3LYP/6-311++G(2d,p) level of theory for ${ }^{1} \mathrm{H}$ and IEF-PCM/MPW1PW91/6- 311+G(2d,p) level of theory for ${ }^{13} \mathrm{C}$ chemical shifts. Use of the method based on proton shift comparisons is recommended for compounds of this type because it showed better ability to discriminate between trans and cis isomers differences than did the carbon-based method.

\section{Experimental Section}

General. ${ }^{1} \mathrm{H}$ and ${ }^{13} \mathrm{C}$ NMR spectra were recorded on a Varian Unity Inova NMR spectrometer at $300 \mathrm{MHz}$ operating at a proton frequency of $299.75 \mathrm{MHz}$ and $75.37 \mathrm{MHz}$ for carbon in $d^{4}$ methanol with TMS as an internal standard. ${ }^{1} \mathrm{H}$ NMR spectra were acquired with 16 scans, $5999.25 \mathrm{~Hz}$ spectral width, and $0.8 \mathrm{~s}$ relaxation decay. Data were zerofilled up to $32 \mathrm{k}$, apodized with $0.1 \mathrm{~Hz}$ exponential line broadening function and Fourier transformed, auto phased by baseline optimization using ACDLabs SpecManager 4.0 software. ${ }^{22}{ }^{1} \mathrm{H}$ NMR shifts were assigned using the coupling constant analysis and ${ }^{1} \mathrm{H},{ }^{1} \mathrm{H}-\mathrm{COSY}$ experiments. Mass spectra were recorded on the Quattro Premier XE tandem quadrupole mass spectrometer (Waters, Manchester, UK) and relative abundances of fragments are quoted in parentheses after the $\mathrm{m} / \mathrm{z}$ values. The IR spectra were recorded on the Perkin-Elmer Spectrum GX FT-IR spectrometer. The compounds were identified by the microanalysis in the Analytical Laboratory at Faculty of Chemistry of Vilnius University. HPLC analysis was performed as described in the literature. ${ }^{\text {Error! Bookmark not }}$ defined. 


\section{Typical experimental procedure for the PT catalysts promoted 4-methylpyridinium salt cyclization reaction with dibromides}

To a solution of 1-ethyl-4-methylpyridinium bromide $(25 \mathrm{mmol})$ and dibromide $\mathbf{2 a - c}(75 \mathrm{mmol})$ in $50 \mathrm{ml}$ dry acetonitrile $25 \mathrm{mmol}$ of the one of the next PT catalyst: 15-crown-5, dicyclohexyl18-crown-6, TEBA-Cl or ethyltriphenylphosphonium bromide was added. The reaction mixture was heated, with vigorously stirring, to $\sim 65{ }^{0} \mathrm{C}$ and $10.35 \mathrm{~g}(75 \mathrm{mmol})$ of anhydrous potassium carbonate ( $1 \mathrm{~g}$ every $30 \mathrm{~min}$ ) was added. After the addition was complete, the reaction mixture was heated 2 hour and then allowed to reach the room temperature and leaved overnight. Next day the mixture was filtered, a residue was treated with $250 \mathrm{ml}$ of acetonitrile and inorganic salts were filtered off. Filtrate was concentrated under vacuum. This crude reaction mixture was purified by flash silica gel column chromatography $\left(\mathrm{CH}_{3} \mathrm{CN} / \mathrm{CHCl}_{3}\right.$ solvent systems).

1-ethyl-4-cyclopropylpyridinium bromide (3a). Hygroscopic sand-coloured crystals (78\% with 15-crown-5). Anal. Calcd. $\mathrm{C}_{10} \mathrm{H}_{14} \mathrm{BrN}$ (228.1): C, 52.65; H, 6.19; Br, 35.03; N, 6.14.

Found: C, 52,37; H, 6.11; N, 6.05. ${ }^{1} \mathrm{H}-\mathrm{NMR}\left(\mathrm{CD}_{3} \mathrm{OD}\right) \delta$ : 1.16-1.22 (m, 2H, H-9b, 10b), 1.44-1.52

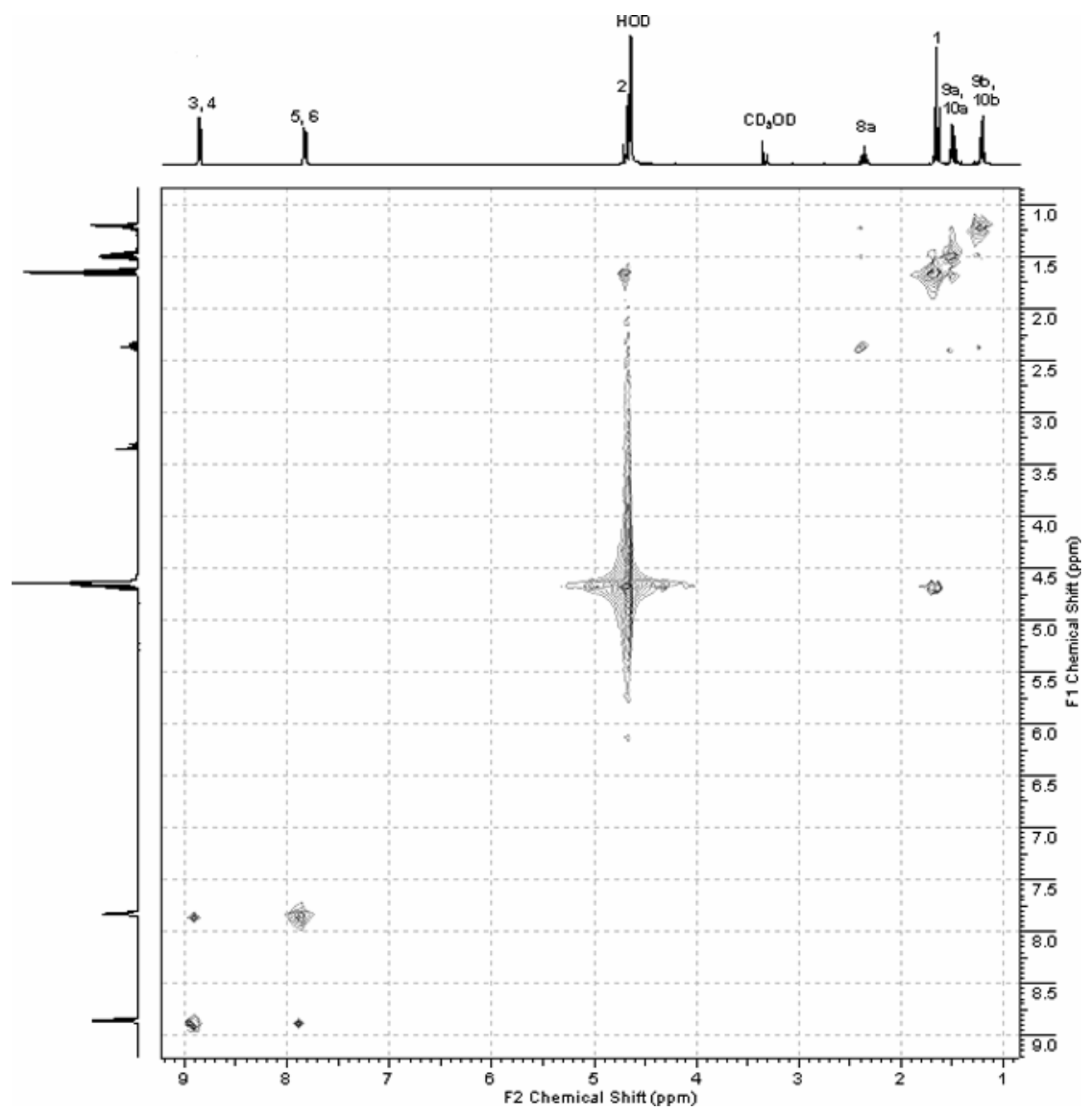

Figure 5. COSY H,H and ${ }^{1} \mathrm{H}$ NMR spectrum of the compound 3a. 
(m, 2H, H-9a,10a), 1.63 (t, $J=7.3 \mathrm{~Hz}, 3 \mathrm{H}, \mathrm{H}-1), 2.30-2.38(\mathrm{~m}, 1 \mathrm{H}, \mathrm{H}-8 \mathrm{a}), 4,63$ (q, $J=7.5 \mathrm{~Hz}$, $2 \mathrm{H}, \mathrm{H}-2), 7.77-7.85$ (m, 2H, H-5,6), 8.79-8.89 (m, 2H, H-3,4). ${ }^{13} \mathrm{C}$ NMR $\left(\mathrm{CD}_{3} \mathrm{OD}\right) \delta: 15.26(\mathrm{C}-$ 1), 16.79 (C-9,10), 17.51 (C-8), 57.08 (C-2), 125.36 (C-5,6), 144.28 (C-3,4), 168.16 (C-7). FTIR (film) $v_{\max } / \mathrm{cm}^{-1}: 3126,3044,3011,2941,2875,1640,1519,1478,1387,1354,1218,1186$, 1175, 1092, 1048. MS m/z (\%): 148(M+,24), 120(100), 118(26), 103(11), 93(38), 91(20), 80(14), $77(15)$.

1-ethyl-4-(2-phenylcyclopropyl)pyridinium bromide (3b). Hygroscopic sand-coloured crystals (53 \% with 15-crown-5). Anal. Calcd. for $\mathrm{C}_{16} \mathrm{H}_{18} \mathrm{BrN}$ (304.2): C, 63.17; H, 5.96; N, 4.60. Found: $\mathrm{C}, 62.92 ; \mathrm{H}, 6.01 ; \mathrm{N}, 4.56 .{ }^{1} \mathrm{H}-\mathrm{NMR}\left(\mathrm{CD}_{3} \mathrm{OD}\right) \delta: 1.62(\mathrm{t}, J=7.3 \mathrm{~Hz}, 3 \mathrm{H}, \mathrm{H}-1), 1.84-1.91(\mathrm{~m}, 1 \mathrm{H}$, H-10a), 1.93-1.99 (m, 1H, H-10b), 2.5-2.56 (m, 1H, H-9b), 2.65-2.72 (m, 1H. H-8a), 4.60 (q, $J=$ $7.3 \mathrm{~Hz}, 2 \mathrm{H}, \mathrm{H}-2)$, 7.18-7.25 (m, 3H, H-14,15), 7.26-7.32 (m, 2H, H-13), 7.81-7.87 (m, 2H, H5,6), 8.75-8.81 (m, 2H, H-3,4). ${ }^{13} \mathrm{C}$ NMR (CD $\left.{ }_{3} \mathrm{OD}\right) \delta: 16.70$ (C-1), 22.65 (C-10), 29.39 (C-8),

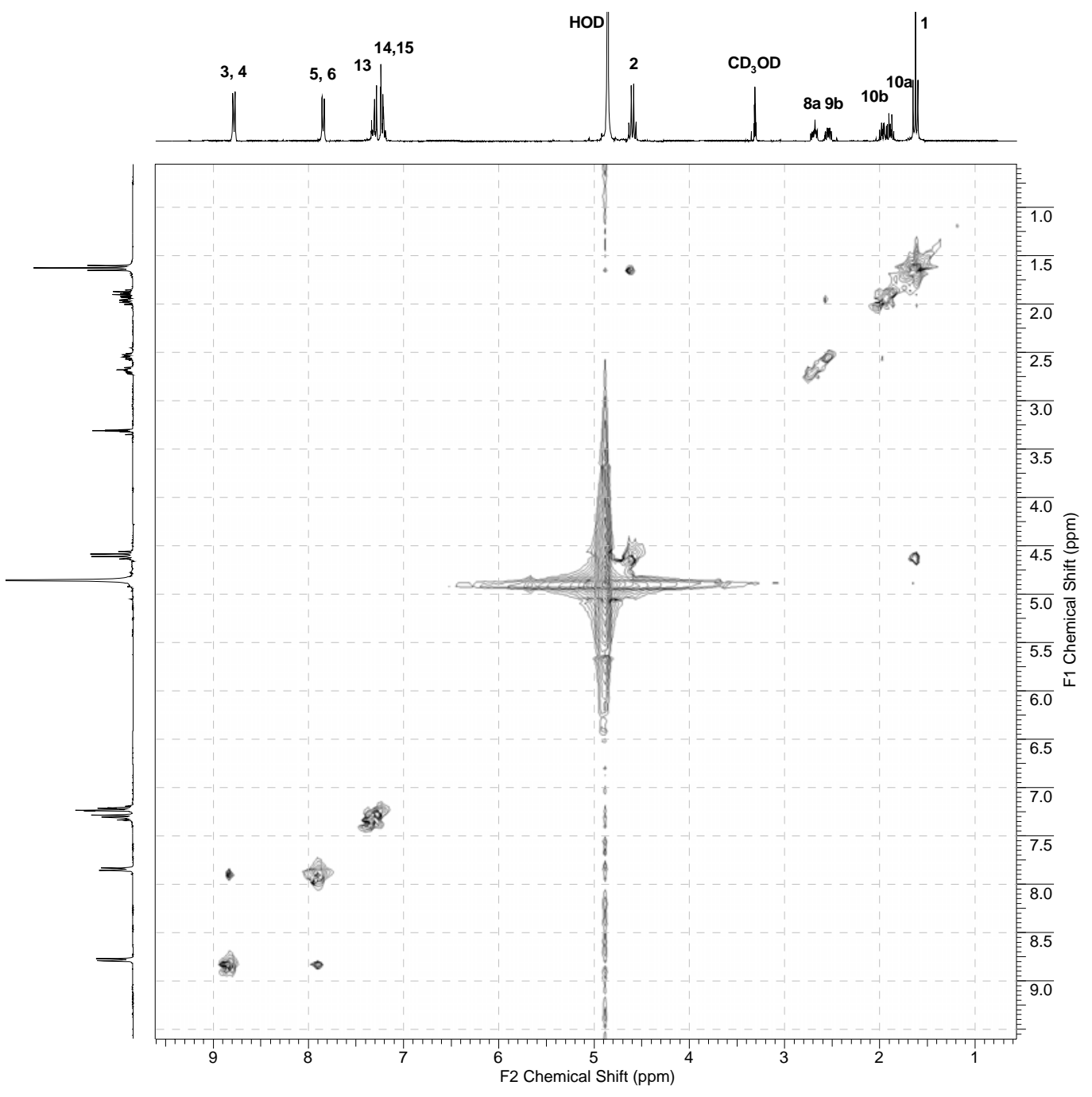


Figure 6. COSY H,H and ${ }^{1} \mathrm{H}$ NMR spectrum of the compound $\mathbf{3 b}$.

34.18 (C-9), 57.21 (C-2), 125.49 (C-5,6), 127.10 (C-14), 127.83 (C-13), 129.66 (C-15), 141.20 (C-12), 144.41 (C-3,4), 165.90 (C-7). FT-IR (film) $v_{\max } / \mathrm{cm}^{-1}: 3122,3122,3030,2935,2872$, $1639,1603,1560,1519,1459,1377,1351,1213,1180,1168,1116,1078 . \mathrm{MS} \mathrm{m} / \mathrm{z}(\%):$ 224(M+,42), 196(100), 194(48), 180(31), 167(16), 154(10), 146(19), 129(42), 118(70), 103(20), 93(21), 91(98), 80(72).

1-Ethyl-4-cyclobutylpyridinium bromide (3c). Hygroscopic sand-coloured crystals (51\% with 15-crown-5). Anal. Calcd. for $\mathrm{C}_{11} \mathrm{H}_{16} \mathrm{BrN}$ (242.2): C, 54.56; H, 6.66; N, 5.78. Found: C, 54.41;

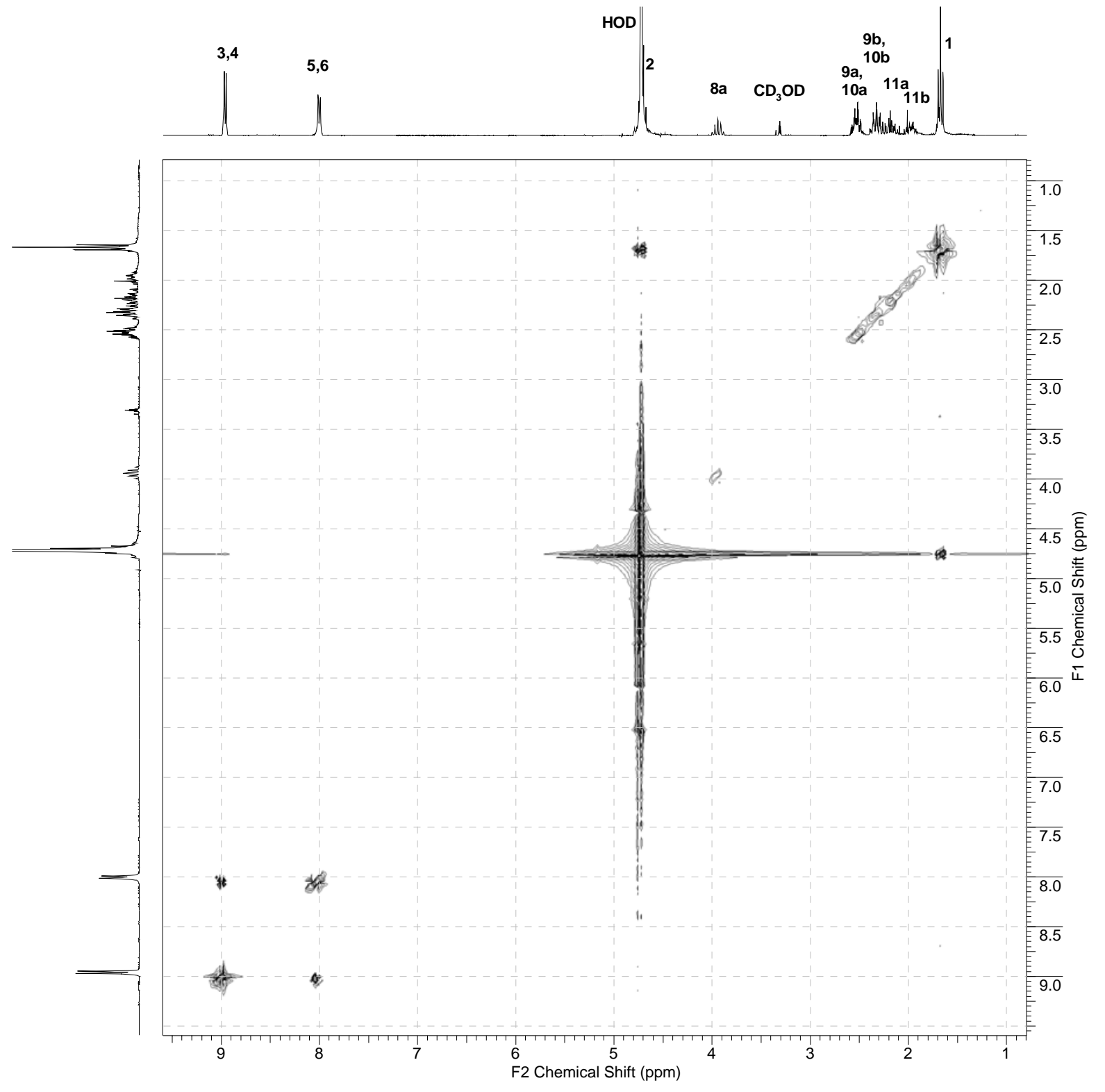

Figure 7. COSY H,H and ${ }^{1} \mathrm{H}$ NMR spectrum of the compound 3c. 
$\mathrm{H}, 6.64$; N, 5.68. ${ }^{1} \mathrm{H}-\mathrm{NMR}\left(\mathrm{CD}_{3} \mathrm{OD}\right) \delta$ : 1.64 (m, $\left.J=7.3 \mathrm{~Hz}, 3 \mathrm{H}, \mathrm{H}-1\right), 1.89-2.01$ (m, 1H, H-11b), 2.10-2.24 (m, 1H, H-11a), 2.23-2.39 (m, 2H, H-9b,10b), 2.46-2.57 (m, 2H, H-9a, 10a), 3.82-4.00 (m, 1H, H-8a), 4.65 (q, $J=7.32 \mathrm{~Hz}, 2 \mathrm{H}, \mathrm{H}-2), 7.92-7.99$ (m, 2H, H-5,6), 8.87-8.93 (m, 2H, H-

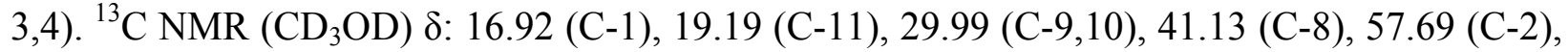
$127.30(\mathrm{C}-5,6), 145.06$ (C-3,4), 167.59 (C-7). FT-IR (film) $v_{\max } / \mathrm{cm}^{-1}: 3119,3035,2977,2940$, 2868, 1639, 1566, 1516, 1470, 1352, 1251, 1174, 1109, 1091. MS m/z (\%): 162(M+,1), 106(100), 79(10), 77(10).

\section{Acknowledgements}

The authors gratefully acknowledge the Lithuanian State Science and Studies Foundation (Project B-38/2008) for financial support. We thank Tomas Petrènas from National food and veterinary risk assessment institute for MS spectrum recording.

\section{References}

1. Todo, Y.; Takagi, H.; Iino, F.; Fukuorka, Y.; Ikeda, Y.; Tanaka, K.; Saikawa, I.; Narita, H. Chem. Pharm. Bull. 1994, 42, 2049. (b) Turner, W. R.; Suto, M. J. Tetrahedron Lett. 1993, 34, 281.

2. Wong, H. N. C.; Hon, M.-Y.; Tse Ch.-W.; Yip, Y.-Ch.; Tank, J.; Hudlicky, T. Chem. Rev. 1989, 89, 165.

3. Ballini, R.; Palmieri, A.; Fiorini, D. Arkivoc 2007, (vii), 172.

4. Marquess, D.; Linsell, M. S.; Turner, S. D.; Trapp, S. G.; Long D. D.; Fatheree, P. R. US Patent 6,878,686, 2005.

5. Lednicer, D.; Mitscher, L. A. In Organic Chemistry of Drug Synthesis; John Wiley: New York, Chichester, Brisbane, Toronto, 1980; Vol. 2, p 31.

6. Gray, A. P.; Kraus, H. J. Org. Chem. 1966, 31, 399.

7. Gray, A. P.; Kraus, H.; Heitmeier, D. E.; Shyley, R. H. J. Org. Chem. 1968, 33, 3007.

8. Levine, R.; Patrick, G. R. J. Org. Chem. 1973, 38, 3942.

9. Eisch, J. J.; Gopal, H.; Russo, D. A. J. Org. Chem. 1974, 39, 3110.

10. Wallace, D. J.; Chen, C. Tetrahedron Let., 2002, 43, 6987.

11. Hall, L.; Murray, S.; Castagnoli, K.; Castagnoli, N. Chem. Res. Toxicol., 1992, 5, 625.

12. Stewart, J. M.; Westberg, H. H. J. Org. Chem. 1965, 30, 1951.

13. Jonczyk, A.; Serafin, B.; Makosza, M. Roczn. Chem. 1971, 45, 2097.

14. Singh, R.K.; Danishefsky, S. J. Org. Chem. 1975, 40, 2969.

15. Butkene, R. V.; Mikulskene, G. V.; Eikher-Lorka, O. S.; K. Kupyatis, G.-K. Chem. Heter. Comp. 1989, 25, 433. 
16. Shen, Q.; Wells, C.; Traetteberg, M.; Bohn, R.K.; Willis, A.; Knee, J. J. Org. Chem. 2001, 66,5840 .

17. Traetteberg, M.; Rauch, K.; de Meijere A. J. Mol. Struct. 2005, 738, 25

18. Frisch, M. J.; Trucks, G. W.; Schlegel, H. B.; Scuseria, G. E.; Robb, M. A.; Cheeseman, J. R.; Montgomery, J. A.; Vreven, Jr. T.; Kudin, K. N.; Burant, J. C.; Millam, J. M.; Iyengar, S. S.; Tomasi, J.; Barone, V.; Mennucci, B.; Cossi, M.; Scalmani, G.; Rega, N.; Petersson, G. A.; Nakatsuji, H.; Hada, M.; Ehara, M.; Toyota, K.; Fukuda, R.; Hasegawa, J.; Ishida, M.; Nakajima, T.; Honda, Y.; Kitao, O.; Nakai, H.; Klene, M.; Li, X.; Knox, J. E.; Hratchian, H. P.; Cross, J. B.; Adamo, C.; Jaramillo, J.; Gomperts, R.; Stratmann, R. E.; Yazyev, O.; Austin, A. J.; Cammi, R.; Pomelli, C.; Ochterski, J. W.; Ayala, P. Y.; Morokuma, K.; Voth, G. A.; Salvador, P.; Dannenberg, J. J.; Zakrzewski, V. G.; Dapprich, S.; Daniels, A. D.; Strain, M. C.; Farkas, O.; Malick, D. K.; Rabuck, A. D.; Raghavachari, K.; Foresman, J. B.; Ortiz, J. V.; Cui, Q.; Baboul, A. G.; Clifford, S.; Cioslowski, J.; Stefanov, B. B.; Liu, G.; Liashenko, A.; Piskorz, P.; Komaromi, I.; Martin, R. L.; Fox, D. J.; Keith, T.; Al-Laham, M. A.; Peng, C.Y.; Nanayakkara, A.; Challacombe, M.; Gill, P. M. W.; Johnson, B.; Chen, W.; Wong, M. W.; Gonzalez, C.; Pople, J. A. In Gaussian, Inc., Pittsburgh PA, 2003.

19. http://www.chemcraftprog.com

20. Sadikov, N.; Nasibov, S.; Öğretir, C.; Berber, H.; Hüseyinli, A. Molecule 2004, 9, 922.

21. Wiitala, K. W.; Cramer, Ch. J.; Hoye, T. R. Magn. Reson. Chem. 2007, 45, 819.

22. Wiitala, K. W.; Al-Rashid, Z. F.; Dvornikovs, V.; Hoye T. R.; Cramer, Ch. J. J. Phys. Org. Chem. 2007; 20, 345.

23. www.acdlabs.com 